\title{
Menopause-related osteoporosis
}

\author{
Leon Snyman \\ Department of Obstetrics and Gynaecology, University of Pretoria, Pretoria \\ Authore-mail: leon.snyman@up.ac.za
}

Peak bone mass for spine and hip is reached in the mid-twenties and adolescents should be counselled on adequate nutrition to ensure sufficient calcium intake, regular weight-bearing exercise, maintaining normal body weight, avoiding smoking and limiting alcohol intake. These measures are important to prevent osteopenia and osteoporosis by obtaining a maximum peak bone mineral density (BMD) and to maintain it by avoiding excessive bone loss. One year before the onset of menopause, however, as a result of oestrogen deficiency, there is an increase in osteoclastic activity without a similar increase in osteoblastic activity, resulting in accelerated bone loss. The average decrease in BMD during the menopausal transition is estimated to be about $10 \%$ and a woman's risk of sustaining an osteoporotic or fragility fracture doubles for each decade after the age of fifty. Half of women older than 50 years of age will be osteopenic compared to $10 \%$ who are osteoporotic and only $40 \%$ will have normal BMD. This article gives an overview of the prevention, diagnosis and management of osteoporosis during and before menopause.

Keywords: fragility fractures, menopause, osteoporosis

\section{Introduction}

A higher percentage of women is living longer in certain communities. In the UK, it is estimated that $13 \%$ of women born in 1951 will be alive in 2051, and an estimated 40\% of girls born in 2013 will celebrate their $100^{\text {th }}$ birthday. This increase in life expectancy has also been reported in other countries, and should result in many women living through the postmenopausal stage of their lives for a further 50 years. ${ }^{1}$ Consequently, menopause-related osteoporosis will be a major health concern for these women and their healthcare providers.

Low bone mineral density (BMD) is responsible for a growing global health burden. Increased rates of age-standardised disability adjusted life years attributable to low body mass index have been observed in developing regions, such as subSaharan, East and West Africa. ${ }^{2}$ A woman's risk of sustaining an osteoporotic or fragility fracture increases with age, and the risk doubles for each decade after the age of 50 . There are serious implications following hip and vertebral fractures in older women. Fragility fractures result in pain, hospitalisation, loss of function and independence. More than half of women who have sustained hip fractures are unable to climb stairs, get in and out of the shower, or use a toilet without assistance one year after surgery. Mortality attributable to surgery following hip fractures is estimated to be approximately $4 \%$, and roughly $20 \%$ of these patients will die within a year of sustaining a hip fracture. $^{3}$

\section{Pathophysiology and diagnosis}

Peak bone mass for the spine and hip is reached in the mid-20s. One year before the onset of menopause, there is an increase in osteoclastic activity without a similar increase in osteoblastic activity, resulting in accelerated bone loss as a result of oestrogen deficiency. The average decrease in BMD during the menopausal transition is estimated to be about $10 \%$. In addition to $\mathrm{BMD}$, bone quality also contributes to bone strength. ${ }^{4}$

Risk factors for developing osteoporosis include age, gender, race, genetics and dietary calcium intake. Lifestyle issues, such as smoking, exercise, alcohol consumption and sunlight exposure, also contribute to this risk. ${ }^{4}$

Osteoporosis is diagnosed in menopausal women when the $\mathrm{BMD}$ assessment by dual-energy $\mathrm{X}$ ray absorptiometry is less than or equal to 2.5 standard deviations below that of the young adult reference population. This value is known as the $T$-score. According to the World Health Organization (WHO) diagnostic guidelines, a $T$-score of +2.5 to -1 is normal, a $T$-score between -1.0 and -2.5 is osteopenia, and if the $T$-score is lower than -2.5 , the individual is diagnosed as having osteoporosis. Osteoporosis is diagnosed clinically in the presence of a fragility fracture regardless of the $T$-score. A fragility fracture is defined as a fracture resulting from a low trauma event, such as falling from a standing height or less. ${ }^{5}$

The abovementioned diagnostic criteria are not applicable to healthy premenopausal women. The Z-score compares an individual's BMD to that of an age-matched population, and this score is used in the diagnosis of osteoporosis in this group of women. A Z-score of more than 2 below the standard deviation of the mean should prompt investigations to diagnose underlying causes of bone loss. ${ }^{5}$

A thorough history and physical examination is imperative to rule out secondary causes of osteoporosis in postmenopausal women. Special investigations that can be performed include full blood count, sedimentation rate, C-reactive protein level, serum calcium, albumin, creatinine, phosphate, alkaline phosphatase, liver transaminases and thyroid function tests. Further appropriate special investigations are indicated depending on the information obtained from the history and an examination, where possible, if secondary osteoporosis is suspected. If a secondary cause is not suspected, it is probably not necessary to perform additional special investigations. Table I provides a list of conditions that can cause secondary osteoporosis.

More elderly women with osteopenia will sustain fragility fractures than women with osteoporosis because of a higher 
Table I: Causes of secondary osteoporosis

\begin{tabular}{l} 
Medical conditions \\
\hline Eating disorders \\
Gastrointestinal malabsorption \\
Vitamin D and/or calcium deficiency \\
Hyperthyroidism \\
Hyperparathyroidism \\
Cushing's syndrome \\
Hypogonadism \\
Chronic inflammatory conditions \\
Alcoholism \\
Renal disease \\
Liver disease \\
Osteogenesis imperfecta \\
Marfan's syndrome \\
Hereditary haemochromatosis \\
Thalassemia major \\
Gaucher's disease \\
HIV infection and/or medications \\
Diabetes mellitus \\
Thalassemia major \\
Medications \\
Glucocorticoids \\
Immunosuppressant drugs \\
Antiepileptic drugs (phenobarbital and phenytoin) \\
GnRH agonists \\
Heparin \\
Chemotherapy, causing amenorrhea \\
Depot medroxyprogesterone acetate
\end{tabular}

GnRH: gonadotrophin-releasing hormone

HIV: human immunodeficiency virus

number of women in the osteopenia group. Half of women older than 50 years will be osteopenic compared to $10 \%$ who are osteoporotic and $40 \%$ who have normal BMD. Women with osteopenia need to be assessed and managed for fracture risk using modalities such as the Fracture Risk Assessment Tool $\left(\right.$ FRAX $^{\circledR}$ ) which was developed by the WHO. ${ }^{6}$ Use of the FRAX ${ }^{\circledR}$ assessment tool is hampered by the limited availability of epidemiological fracture data from South Africa.

\section{Prevention}

Prevention of osteoporosis starts early in life, and adolescents should be counselled with regard to adequate nutrition, regular exercise and the promotion of healthy lifestyle issues, such as achieving a normal body weight, avoiding smoking and ensuring moderate alcohol intake.,8 These measures are important in obtaining maximum peak BMD, as well as maintaining it by avoiding excessive bone loss.

Calcium supplementation has been widely advocated as an effective preventative measure against osteoporosis for many years, and supplementation from an early age advised in an effort to ensure maximum peak bone mass in the third decade of life. Recent meta-analyses suggest that benefit is not derived from calcium supplementation, with or without vitamin D supplementation, in the prevention of postmenopausal vertebral, non-vertebral or hip fractures in women living in communities. ${ }^{9}$ It is concerning that some data suggest that calcium supplementation is associated with an increased risk of myocardial infarction, strokes, the formation of kidney stones and hospitalisation for acute gastrointestinal problems..$^{10,11}$

The preferred way of obtaining adequate calcium is through the diet, and calcium supplementation is not recommended in women who have an adequate dietary calcium intake. Adequate daily dietary calcium intake has not been associated with the same adverse risks associated with calcium supplementation. The daily recommended intake of calcium through the diet is 1 000-1 $200 \mathrm{mg}$ per day and $600-800 \mathrm{IU}$ of vitamin D. A glass of full cream milk contains approximately $300 \mathrm{mg}$ of calcium. Other calcium-containing foods include dark, leafy greens, cheese, yoghurt fish and almonds. Fatty fish, such as tuna and salmon, and beef liver, eggs and cheese contain high levels of vitamin D.

Preventative pharmacological intervention, aimed at minimising bone loss and preventing fractures, should be individualised after clinical information, possible benefits, and the costs and risks attributable to different medications have been taken into consideration.

\section{Screening}

There are no universally accepted screening practices for osteoporosis due to unresolved issues with regard to cost and effectiveness of screening. Screening involves identifying patients at risk of sustaining fragility fractures, emphasising the importance of taking a complete and thorough history aimed at identifying these risk factors. Besides BMD, a history of a previous fragility fracture and advanced age are the most robust risk factors predicting fragility fracture risk.

The recommendations from different expert groups vary considerably. The United States Preventive Services Task Force (USPSTF) recommends BMD assessment in all post-menopausal women older than 65 years, and in younger women with one or more risk factors and a history of a previous fragility fracture. ${ }^{4}$ The National Osteoporosis Foundation (NOF) recommends BMD assessment in women between 50 and 70 years with risk factors. In the absence of formal recommendations, screening should be individualised and offered to post-menopausal and other women at risk for sustaining fragility fractures.

\section{Management}

Management of women with low and moderate fracture risk mainly centres on prevention. These women should be counselled with regard to healthy lifestyle measures and adequate dietary calcium and vitamin D intake. Besides a diet that ensures adequate calcium, vitamin D and protein intake, other nonpharmacological interventions include regular weight-bearing exercise and exercises aimed at strengthening muscles to reduce the risk of falls. Additional fall-prevention strategies, such as home safety assessment and modification interventions, replacing multifocal with single lenses, as well as a prescribing modification programme from primary care physicians, have been shown to significantly reduce the risk of falls in elderly community-dwelling individuals. ${ }^{12}$

In addition to the above, pharmacological treatment should be considered in women with a high fracture risk. Women with $T$-scores below -2.5 , and those with previous osteoporotic fractures are at high risk of osteoporotic fractures and might benefit from pharmacological intervention. The latter might be indicated in osteopenic women with a 10-year probability risk of hip fracture of $3 \%$ or more or a $20 \%$ probability risk of sustaining a major osteoporotic fracture. ${ }^{5}$

\section{Available preparations Hormone therapy}

Menopausal hormone therapy (MHT) in the form of oestrogen, with or without progestin, is very effective in increasing 
$B M D$, and more importantly, in reducing clinical fracture risk. Oestrogen decreases hip and other fractures by $30-50 \%{ }^{4}$ Hormone therapy should be the treatment of choice in women with osteoporosis aged 40-50 years, who do not have contraindications, and especially in women with menopausal symptoms. Hormonal treatment should not be initiated after the age of 60 years. Continued use after this age must be individualised. Extending MHT to individual women is acceptable practice when the benefits outweigh potential risks. Hormone therapy should be prescribed at a dose known to provide fracture risk reduction, which is $0.625 \mathrm{mg} /$ day of conjugated equine oestrogen, or equivalent doses of other preparations. $^{13}$

The protective effect on BMD and fracture risk is lost within two years of the cessation of hormone therapy. Therefore, it is important to reassess BMD and fracture risk in women who stop hormone therapy and to consider other treatment options when indicated. ${ }^{14}$

\section{Bisphosphonates}

Bisphosphonates act on an enzymatic level, reducing osteoclastic activity in bone, which results in the suppression of bone turnover and preservation of the bone architecture. Preparations include alendronate (Fosamax ${ }^{\circledR}$ ), ibandronate (Bondronat ${ }^{\oplus}$ ), risedronate $\left(\right.$ Actonel $\left.^{\oplus}\right)$ and zoledronic acid (Aclasta $\left.{ }^{\oplus}\right)$. Alendronate combined with cholecalciferol (Fosavance ${ }^{\oplus}$ ) can be administered weekly. Bisphosphonates are frequently used as first-line pharmacological treatment for menopause-related osteoporosis.

These preparations are effective in reducing fracture risk. Alendronate is associated with a relative risk reduction of $47 \%$ for vertebral fractures and $51 \%$ for hip fractures. The respective figures for risedronate are $41 \%$ and $30 \%$, while $5 \mathrm{mg}$ of zoledronic acid, infused yearly for three years, reduces vertebral fractures by $70 \%$ and hip fractures by $41 \% .^{15}$

Oral bisphosphonates are poorly absorbed and must be taken on an empty stomach. Patients should not lie down for at least 30 minutes after ingestion. Oesophageal irritation is a common side-effect. Osteonecrosis of the jaw and atypical fractures are rare complications of bisphosphonate use in women treated for osteoporosis. $^{13}$

Bisphosphonate treatment should be discontinued after 5-10 years, depending on the original clinical state and response to treatment. After the cessation of bisphosphonate therapy, BMD measurements, with or without bone turnover markers, should be performed every two years. Therapy can be recommenced if there is a consistent decrease in BMD.

\section{Selective oestrogen-receptor modulators}

Raloxifene (Evista ${ }^{\oplus}$ ) acts like an oestrogen agonist in bone tissue, and as an antagonist on endometrial and breast tissue. It reduces vertebral fractures by $30 \%$, and increases BMD significantly in the vertebra and the hip, but does not reduce the risk of nonvertebral fractures. It is contraindicated in women with previous venous thromboembolism (VTE), and does not relieve menopausal vasomotor symptoms. ${ }^{15,16}$

\section{Teriparatide}

This preparation contains recombinant human parathyroid hormone. It stimulates bone formation and the remodelling of bone, resulting in an increase in BMD and an improvement in bone architecture. It significantly increases BMD, and reduces vertebral fractures by $65 \%$ and non-vertebral fractures by $53 \%$. BMD decreases rapidly after the cessation of therapy. ${ }^{17}$

Side-effects include hypercalcaemia, hypercalciuria, nausea and headaches. Teriparatide is indicated in cases of failed antiresorptive therapy, severe fracturing disease and osteoporosis secondary to glucocorticoid use. Treatment duration should not exceed 18 months. ${ }^{13}$

\section{Strontium ranelate}

Strontium ranelate inhibits bone resorption and increases bone formation. The reduction in vertebral fractures is $37 \%$ after $2 \mathrm{~g} /$ day over three years, and for the same dosage over the same time, $14 \%$ for non-vertebral fractures. Side-effects include diarrhoea, as well as potential vascular and nervous system side-effects. ${ }^{18}$ It should not be used in patients with peripheral vascular or cardiovascular disease, uncontrolled hypertension or women at risk of VTE. ${ }^{13}$

\section{Monitoring therapy}

There is no consensus as to what constitutes the optimal monitoring of patients being treated for menopause-related osteoporosis. The main reason for monitoring these patients is to identify patients who require a change in therapy.

Patients on treatment should be monitored clinically to exclude serious side-effects from drug treatment and the presence of new fractures.

Most guidelines recommend repeated BMD examinations to be performed every 1-2 years. An increase in BMD is associated with decreased fracture risk. Fracture risk is also decreased if the BMD remains stable. ${ }^{19}$ BMD changes of less than $2-3 \%$ in the spine and $5-6 \%$ at the hip may be as a result of precision error.

There is inefficient evidence with regard to the choice of bone turnover markers to be used in routine clinical practice to monitor osteoporosis treatment response. ${ }^{20}$

\section{Conclusion}

Menopause-related osteoporosis remains an important women's health issue, especially in healthy populations, and as an increasing number of women are living longer. Nonpharmacological interventions, such as adequate dietary calcium and vitamin D intake, smoking cessation, exercise and moderate alcohol consumption, are all effective in the prevention and treatment of osteoporosis.

MHT is still a safe and effective treatment option in women with osteoporosis and those with a high risk of fragility fracture. Bisphosphonates are a safe and effective first-line treatment option, and several other pharmacological interventions are available for poor responders or women with other clinical factors or contraindications.

\section{References}

1. Appleby J. How long can we expect to live? BMJ. 2013;346:f331.

2. Sanchez-Riera L, Carnahan E, Vos T, et al. The global burden attributable to low bone mineral density. Ann Rheum Dis. 2014 (E-pub ahead of print].

3. Friedman SM, Mendelson DA. Epidemiology of fragility fractures. Clin Geriatr Med. 2014;30(2):175-181.

4. Tella SH, Gallagher JC. Prevention and treatment of postmenopausal osteoporosis. J Steroid Biochem Mol Biol. 2013 [E-pub ahead of print].

5. NOF's clinician's guide to prevention and treatment of osteoporosis. National Osteoporosis Foundation [homepage on the Internet]. 2013. c2014. Available from: http://nof.org/hcp/clinicians-guide. 
6. Zhang J, Morgan SL, Saag KG. Osteopenia: debates and dilemmas. Curr Rheumatol Rep. 2013;15(12):384.

7. Howe TE, Shea B, Dawson LJ, et al. Exercise for preventing and treating osteoporosis in postmenopausal women. [Cochrane review]. In: The Cochrane Library, Issue 7; 2011. Oxford: Update Software.

8. Bielemann RM, Martinez-Mesa J, Gigante DP. Physical activity during life course and bone mass: a systematic review of methods and findings from cohort studies with young adults. BMC Musculoskelet Disord. 2013;14:77.

9. Murad MH, Drake MT, Mullan RJ, et al. Clinical review. Comparative effectiveness of drug treatments to prevent fragility fractures: a systematic review and network meta-analysis. J Clin Endocrinol Metab. 2012;97(6):1871-1880.

10. Reid IR. Should we prescribe calcium supplements for osteoporosis prevention? J Bone Metab. 2014;21(1):21-28.

11. Reid IR, Bolland MJ. Calcium risk-benefit updated: new WHI analyses. Maturitas. 2014;77(1):1-3.

12. Gillespie LD, Robertson MC, Gillespie WJ, et al. Interventions for preventing falls in older people living in the community. [Cochrane review]. In: The Cochrane Library, Issue 9, 2012. Oxford: Update Software.

13. Hough FS, Brown SL, Cassim B, et al. The safety of osteoporosis medication. S Afr Med J 2014;104(4)279-282.

14. Karim R, Dell RM, Greene DF, et al. Hip fracture in postmenopausal women after cessation of hormone therapy: results from a prospective study in a large health management organization. Menopause. 2011;18(11):1172-1177.
15. Reginster JY. Antifracture efficacy of currently available therapies for postmenopausal osteoporosis. Drugs. 2011;71(1):65-78.

16. Riggs BL, Hartmann LC. Selective estrogen-receptor modulators: mechanisms of action and application to clinical practice. N Engl J Med. 2003;348(7):618-629.

17. Neer RM, Arnaud CD, Zanchetta JR, et al. Effect of parathyroid hormone (1-34) on fractures and bone mineral density in postmenopausal women with osteoporosis. N Engl J Med. 2001;344(19):1434-1441.

18. O'Donnell S, Cranney A, Wells GA, et al. Strontium ranelate for preventing and treating postmenopausal osteoporosis. [Cochrane review]. In: The Cochrane Library, Issue 4, 2006. Oxford: Update Software.

19. Hochberg MC, Ross PD, Black $D$, et al. Larger increases in bone mineral density during alendronate therapy are associated with a lower risk of new vertebral fractures in women with postmenopausal osteoporosis. Fracture Intervention Trial Research Group. Arthritis Rheum. 1999;42(6):1246-1254.

20. Burch J, Rice S, Yang H, et al. Systematic review of the use of bone turnover markers for monitoring the response to osteoporosis treatment: the secondary prevention of fractures, and primary prevention of fractures in high-risk groups. Health Technol Assess. 2014;18(11):1-180. 